\section{Räumliche Ansprüche des Sektors „Freizeit und Touris- mus" an Natur und Landschaft}

\author{
von Barbara Engels, Bundesamt für \\ Naturschutz
}

Freizeit und Tourismus stellen verschiedenste Nutzungsansprüche an Natur und Landschaft. Während die quantitative Flächeninanspruchnahme aufgrund der Vielzahl der mit Tourismus und Freizeit verbundenen Aktivitäten schwer abzuschätzen ist, liegen über qualitative Nutzungsansprüche aussagekräftige Daten vor. Das wachsende Interesse an Tourismus- und Freizeitangeboten in deutschen Schutzgebieten stellt für das Management dieser Gebiete neue Herausforderungen dar. Internationale und nationale Richtlinien und Zielsetzungen sowie praktisch anwendbare Managementkonzepte bieten den Rahmen für eine nachhaltige touristische Nutzung, welche modernen Nutzungsansprüchen gerecht werden kann.

\section{Touristische Flächennutzung in Deutschland}

Viele Formen des Tourismus sind im Hinblick auf die Flächennutzung relevant; dazu zählen insbesondere auch sport- und naturtouristische Aktivitäten. Der Blick auf die Entwicklung des Tourismus in Deutschland zeigt, dass in den vergangenen zehn Jahren Gästeankünfte und Übernachtungszahlen stetig anstiegen (siehe Tab. 1). Die jährlichen Reiseanalysen der Forschungsgemeinschaft Urlaub und Reisen zeigen zudem, dass Deutschland als Urlaubsreise- ziel der Deutschen mit 31,4 Prozent aller Urlaubsreisen im Jahr 2007 eine besondere Rolle zukommt. Damit fanden 2007 19,5 Mio. Urlaubsreisen der Deutschen in Deutschland statt, eine Zahl die trotz immer billiger werdender Flug- und Fernreisen in den vergangenen Jahren gleich geblieben ist (F.U.R. 2008). Gegenüber 29 Prozent im Jahr 2001 ist der Anteil sogar leicht gestiegen (F.U.R. 2002).

Tourismus ist in zweierlei Hinsicht für die Flächennutzung relevant: $\mathrm{Zu}$ unterscheiden sind dabei flächenverbrauchende und nicht-flächenverbrauchende Aktivitäten.

Die Flächeninanspruchnahme durch den Tourismus umfasst sehr verschiedene Bereiche. Dazu gehören z. B. die Beherbergung, Gastronomie, Verkehrsinfrastruktur, Erholungsflächen sowie Freizeit-, Spiel- und Sportanlagen. Zusätzlich wird ein großer Teil der touristischen Infrastruktur auch und zum Teil sogar überwiegend durch einfache Freizeitaktivitäten oder solche Aktivitäten genutzt, die nicht mit dem Tourismus verbunden sind. Daher gibt es in Deutschland keine umfassende Statistik über die Flächeninanspruchnahme durch den Tourismus (Umweltbundesamt 2002). Die stärkste Auswirkung auf die Flächennutzung hat die touristische Infrastruktur für Beherbergung. Das Öko-Institut errechnete den Flächenbedarf für Beherbergung von Touristen in Deutschland basierend auf der existierenden Bettenkapazität und dem spezifischen Flächenbedarf für Hotellerie, Parahotellerie ${ }^{1}$ und Campingplätze (Umweltbundesamt 2002, S. 28f.) Aus der amtlichen Statistik lässt sich entnehmen, dass sich die Beherbergungskapazität im Vergleich zu 2002 nicht wesentlich erhöht hat. So ist von einem Flächenverbrauch von ca. 27.800 ha auszugehen; das entspricht 0,7 Prozent der gesamten Siedlungsund Verkehrsfläche in Deutschland.

Das Umweltbundesamt kommentiert die-

Tab. 1: Gästeankünfte und -übernachtungen in Deutschland in Beherbergungsstätten und auf Campingplätzen (in Mio.)

\begin{tabular}{lccccccccc}
\hline & 1997 & 2000 & 2001 & 2002 & 2003 & 2004 & 2005 & 2006 & 2007 \\
\hline Ankünfte & 98,1 & 113,7 & 112,8 & 111,0 & 112,8 & 116,4 & 120,6 & 125,2 & 129,9 \\
Übernachtungen & 308,3 & 347,4 & 347,4 & 338,5 & 337,2 & 338,7 & 343,9 & 351,2 & 362,8 \\
\hline
\end{tabular}

Quelle: Statistisches Bundesamt 2008 
sen Wert wie folgt: „Dieser zunächst relativ geringe Wert für Deutschland darf jedoch nicht darüber hinweg täuschen, dass die Umweltbelastungen durch Flächeninanspruchnahme gerade in den bevorzugten Feriengebieten sehr relevant sind. (...) So wird auf den Inseln im Bereich des Nationalparks SchleswigHolsteinisches Wattenmeer über die Hälfte der
Häuser für die Beherbergung von Touristen genutzt.“ (Umweltbundesamt 2002, S. 29) Einen repräsentativen Gesamteindruck zur Verteilung der touristischen Nutzungsintensität als Indikator für den Flächenverbrauch in Deutschland vermitteln die Übernachtungen im Fremdenverkehrsbereich (siehe Abb. 1).

Besonderes touristisches Interesse gilt den

Abb. 1: Übernachtungen im Fremdenverkehr in Deutschland

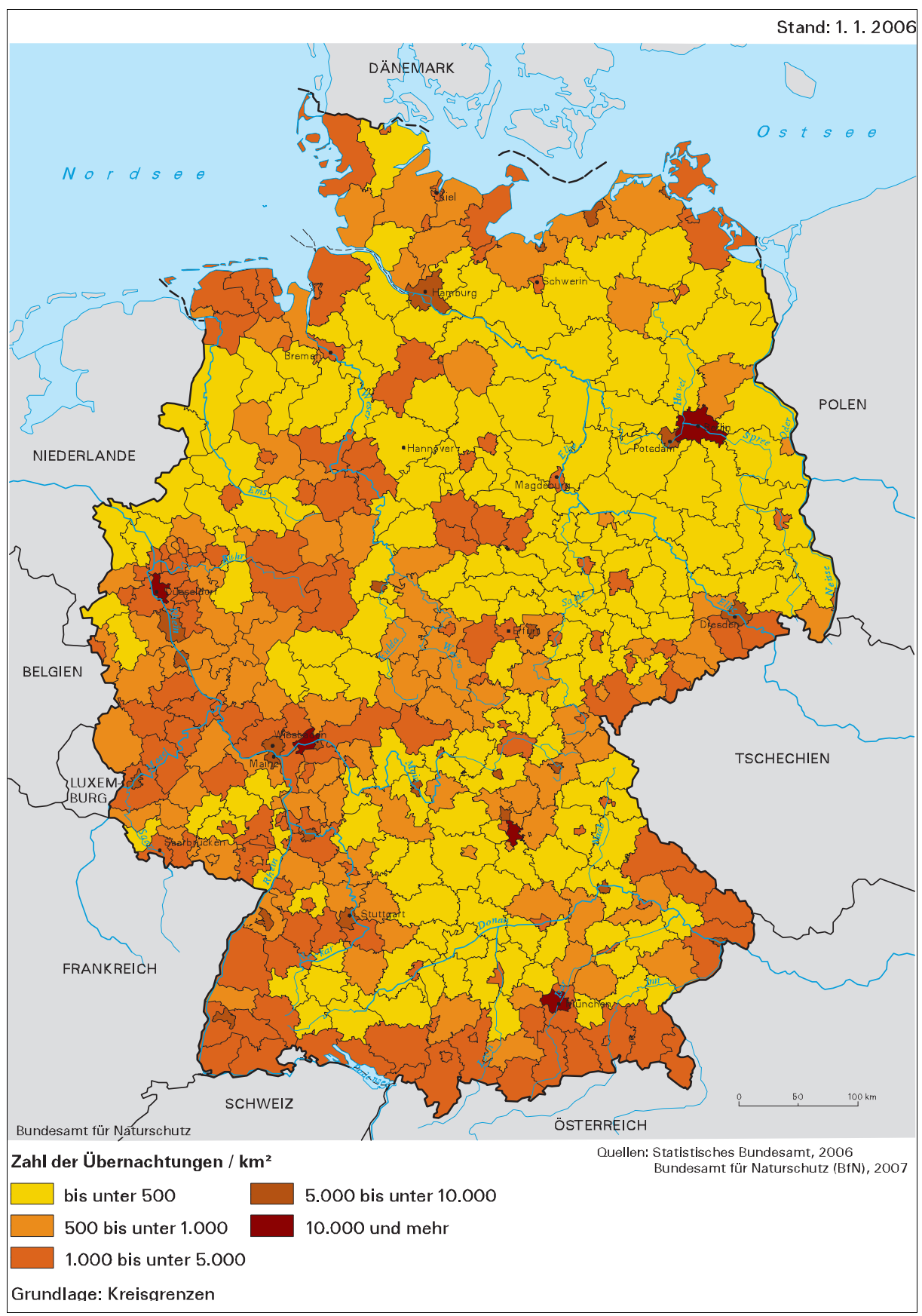

Quelle: Bundesamt für Naturschutz 2008 
Küsten, den Mittelgebirgen einschließlich attraktiver Flusslandschaften und dem Alpenraum. Neben den Großstädten zählen u. a. die an der Küste Mecklenburg-Vorpommerns gelegenen Kreise Stralsund, Rostock und Rügen sowie Lübeck und Ostholstein an der Küste Schleswig-Holsteins $\mathrm{zu}$ den touristisch am intensivsten genutzten Kreisen (mehr als 5.000 Übernachtungen $/ \mathrm{km}^{2}$ ). Dieses spiegelt auch die intensive infrastrukturelle Erschließung des Küstenraumes wider (vgl. Bettenkapazitäten, Statistisches Bundesamt 2007) wider.

$\mathrm{Zu}$ den flächenbeanspruchenden Infrastruktureinrichtungen im Tourismus zählen auch Freizeit- und Erlebnisparks, deren Flächen je nach Anlage zwischen 5 und 300 ha liegenS (vgl. Büro für Tourismus- und Erholungsplanung 1994). So hat etwa der EuropaPark in Rust insgesamt 70 ha Fläche, wovon aber nur 15 Prozent versiegelt sind (Krumrey, Märker 2005). Großflächige Freizeitanlagen sind bezüglich ihrer Kompatibilität mit dem Natur- und Landschaftsschutz umstritten: Einerseits tragen sie dazu bei, die Ströme der Erholungssuchenden zu bündeln, wodurch die freie Landschaft entlastet wird. Andererseits haben sie einen hohen Flächenbedarf und verursachen kleinräumig erhebliche Umweltbelastungen (Verkehr, Abfall, Wasserverbrauch).

\section{Touristische Nachfrage und Flächenqualitäten}

Zentraler als der quantitative Flächenverbrauch, ist die Frage, welche Flächenqualitäten von Tourismus und Freizeit nachgefragt werden und welche sich grundsätzlich für den Tourismus eignen. Dabei sind drei Unterfragen $\mathrm{zu}$ berücksichtigen: 1.) Wie müssen Flächen von der natürlichen Ausstattung her strukturiert sein? 2.) Welche Ausstattung ist notwendig? 3.) Welche Mengen und welcher Typ von touristischer Nachfrage können auf bestimmten abgegrenzten Flächen toleriert werden, ohne andere Nutzungen oder die ökologischen Funktionen des Bodens zu beeinträchtigen (wie z. B. die Tragekapazität der Fläche). ${ }^{2}$

Zur Bewertung der Eignung von Flächen bzw. Landschaften für Freizeit und Tourismus kann z. B. auf den von Kiemstedt 1967 definierten Vielseitigkeitswert zurückgegriffen werden
(Ammer, Pröbstl 1991), der das „Maß der natürlichen Eignung für die Erholung" ausdrückt und neben Wald und Waldrändern, natürliche Gegebenheiten (wie Gewässer, Oberflächengestalt und Nutzungsarten) auch Klima (Niederschlag und Temperatur) mit einbezieht. Diese wissenschaftliche Herangehensweise wurde weiterentwickelt und zunehmend um Kriterien erweitert, welche auch Störfaktoren, die unterschiedlichen Beliebtheiten verschiedener Freizeitaktivitäten, die Erreichbarkeit oder das Vorhandensein von Freizeiteinrichtungen und Sehenswürdigkeiten umfassen (Ammer, Pröbstl 1991).

Auch wenn zunehmend sozioökonomische Kriterien Eingang in die Bewertung der Eignung einer Landschaft für den Tourismus gefunden haben, so bleibt die natürliche Eignung und die vorhandene Infrastruktur entscheidend. Das Bundesamt für Bauwesen und Raumordnung bewertet im Raumordnungsbericht 2005 die touristische und landschaftliche Attraktivität von Regionen mittels eines Indexes, der folgende Indikatoren einschließt: Zerschneidungsgrad, Bewaldungsgrad, Reliefenergie ${ }^{3}$, Wasserflächen und Küstenlinien sowie Übernachtungen im Fremdenverkehr. Dabei wird deutlich, dass insbesondere Küstenregionen und Mittelgebirge sowie Alpenregionen hier am Höchsten bewertet werden (vgl. Abb. 2 nächste Seite). Dies spiegelt sich in der touristischen Nachfrage wider (vgl. Abb. 1).

Vor dem Hintergrund, dass viele der touristischen Infrastruktureinrichtungen bevorzugt in landschaftlich reizvollen und damit häufig auch ökologisch besonders sensiblen Gebieten liegen, kann auch ein mengenmäßig geringer Flächenverbrauch durch den Tourismus gravierende negative Auswirkungen auf die Biodiversität haben (Umweltbundesamt 2002). Dazu zählen z. B. die Zerschneidung von Lebensräumen durch zuleitende Infrastruktur (Verkehrswege) oder der Verlust wesentlicher Habitate. So kann der Bau von Aufstiegshilfen (Skilifte etc.) und Pisten im Alpenraum durch Rodung und Planierung zur Veränderung von Lebensräumen wie Bergwäldern und seltenen artenreichen Bergwiesen führen (Bundesamt für Naturschutz 1997).

Kritisch $\mathrm{zu}$ betrachten ist auch die Entwicklung von neuer touristischer Infrastruktur und insbesondere weiteren Beherbergungskapazitäten vor dem Hintergrund der aktuellen 


\section{Abb. 2: Touristische und landschaftliche Attraktivität}

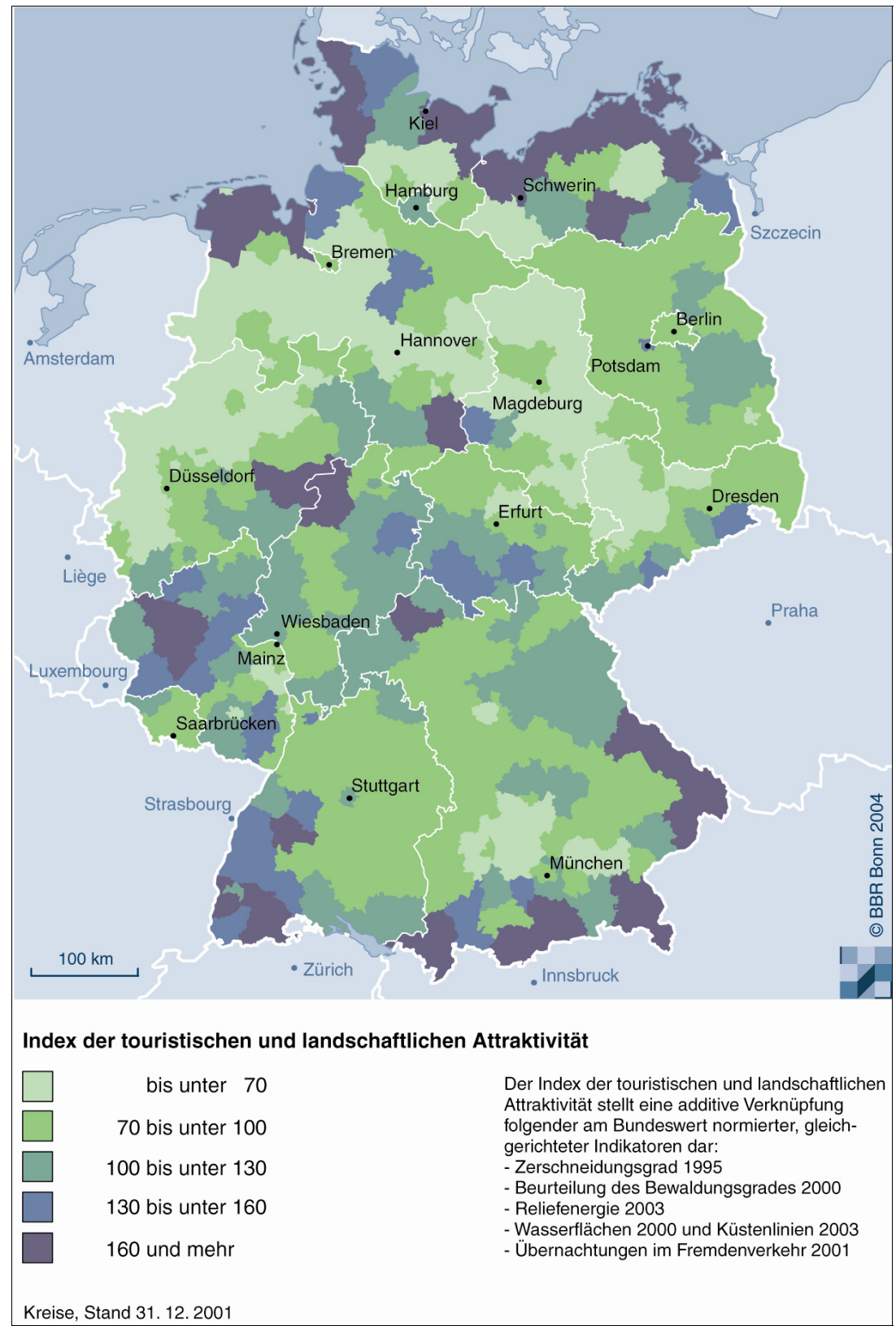

Quelle: BBR 2005 / Laufende Raumbeobachtung des BBR

Auslastung der vorhandenen Kapazitäten. So verzeichnete die amtliche Beherbergungsstatistik im Jahr 2006 eine durchschnittliche Auslastung der verfügbaren bzw. angebotenen Betten von 34,6 bzw. 37,2 Prozent (Statistisches Bundesamt 2007). Ein weiterer Ausbau von Beherbergungskapazitäten (einschließlich des damit verbundenen weiteren Flächen- verbrauchs) ist also auch vor dem Hintergrund ökonomischer Nachhaltigkeit kritisch zu sehen.

Schliephake (1985) beschreibt die Beziehung zwischen Tourismus und Raum als System von Angebot und Nachfrage, bei dem erst die Inwertsetzung und Tragfähigkeit des Raumes (der Landschaft) zur eigentlichen Rentabilität führen. Das Angebot orientiert sich unter Berücksichtigung des natürlichen Potenzials 


\section{Abb. 3: Urlaubslandschaftspräferenzen}

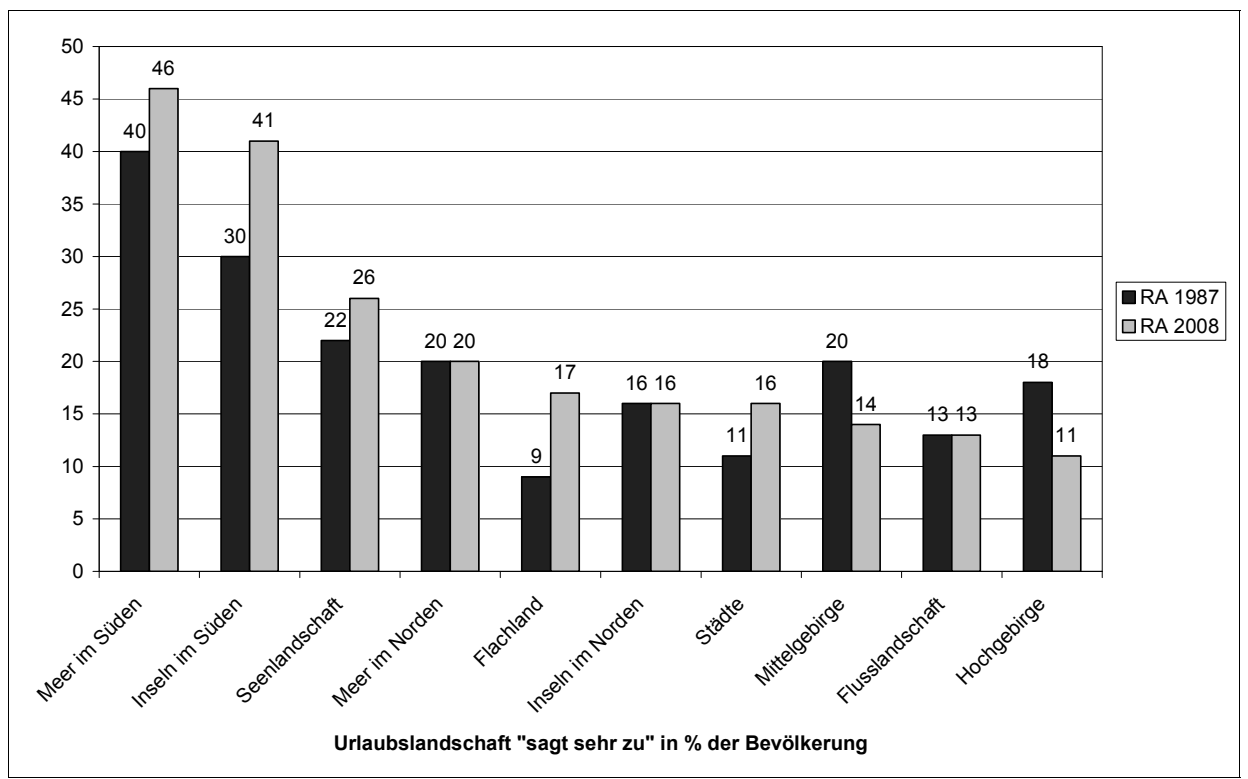

Quelle: F.U.R. 2008

und der Tragfähigkeit an der Nachfrage. Diese wiederum entsteht zum einen in Beziehung zur Kapazität der angebotenen Infrastruktur, zum anderen durch die Bewertung der Attraktivität des Erholungsraumes.

Im Rahmen der Reiseanalyse 2008 wurden die Urlaubslandschaftspräferenzen im Jahr 2007 mit denen vor 20 Jahren verglichen. Dieser Vergleich macht deutlich, dass z. B. in Bezug auf die für Deutschland touristisch relevanten Landschaftstypen, Veränderungen bei der Attraktivität festzustellen sind. Während Hochund Mittelgebirge an Attraktivität verloren haben, so hat das Flachland hinzugewonnen. Gründe hierfür können die zunehmend unsicher werdende Schneesicherheit in tieferen Lagen der Alpen und den Mittelgebirgen, aber auch der demographische Wandel sein: Es gibt immer mehr Senioren in Deutschland und immer mehr von ihnen nehmen am Urlaubsgeschehen teil. In der Altersgruppe der 60- bis 69-Jährigen liegt die Reiseintensität mittlerweile bei 75 Prozent, bei den Über-70-Jährigen bei 60 Prozent (F.U.R. 2006). Urlaubsregionen im Flachland sind für diese Zielgruppe durchaus attraktiv, bieten sie doch ideale Räume für gesundheitsfördernde Aktivitäten wie Radfahren, Nordic Walking und Wandern.

\section{Naturerleben als Tourismustrend}

Diese Veränderungen im Reiseverhalten der Deutschen und damit in der Nachfrage nach touristischen Angeboten haben Auswirkungen auf die Flächennutzung. Aktuelle Trends spielen dabei ebenfalls eine nicht zu vernachlässigende Rolle. So erfreut sich der Gesundheitsund Wellness-Tourismus mit Zunahmen von drei bzw. fünf Prozent im Zeitraum 2004 bis 2006 wachsender Beliebtheit (F.U.R. 2007).

Unterstützt wird dieser Trend durch den Wunsch nach intensivem Erleben von Natur: Für 42 Prozent der Bundesbürgerinnen und bürger zählt „Natur erleben“ zu den wichtigsten Urlaubsmotiven, dessen Bedeutung von 2004 bis 2006 um fünf Prozent zugenommen hat. Dieser Trend hin zu mehr Erholung, Gesundheit und Naturnähe wird seit einigen Jahren durch die spezielle Entwicklung von nachhaltigen natur- und landschaftsorientierten touristischen Aktivitäten in Verbindung mit Naturerlebnis und Gesundheitsaspekten aufgegriffen. Im Mittelpunkt steht dabei verstärkt die Entwicklung von qualitativ hochwertigen touristischen Angeboten, die sich erfolgreich vermarkten lassen (Bundesamt für Naturschutz 2008).

Nationalparke, Naturparke und Biosphärenreservate entwickeln sich dabei zunehmend 
$\mathrm{zu}$ interessanten Destinationen. So geben 60 Prozent der Deutschen an, dass der Besuch eines Nationalparks und Naturerkundungsreisen in Deutschland für sie attraktiv sind $(\mathrm{Ku}-$ ckartz et al. 2006). Diese Tendenz hin zu mehr Freizeit in und mit der Natur bedeutet aber auch ein erhöhtes Gästeaufkommen in ökologisch sensiblen Gebieten und damit einen weiter steigenden Druck auf diese Landschaften und der damit verbundenen Flächennutzung.

Naturtourismus lebt vom Erlebniswert von „Wildnis“ und unberührter Natur, die sich insbesondere in Schutzgebieten finden lässt, er braucht aber auch die umgebende und zuleitende Infrastruktur. Der naturverbundene, individuelle „Ökotourist", der ohne Infrastruktur auskommt, stellt zum einen eine Minderheit dar, zum anderen ist er für den Tourismus als Wirtschaftssektor nicht interessant, da er die touristischen Angebote nicht konsumiert.

\section{Tourismus in ökologisch sensiblen Gebieten}

Insbesondere die insgesamt vierzehn Nationalparke, die mit ihren Attraktionen von Natur und Wildnis ein „knappes Gut" darstellen", nehmen hier eine Sonderstellung ein. Ihre Anzahl ist nicht beliebig vermehrbar, da aufgrund der gesetzlich festgelegten Anforderungen an Nationalparke nur wenige Gebiete in Deutschland die entsprechende Eignung aufweisen (Job 2008). Die derzeit stetig wachsende Attraktivität der bestehenden Parke und die steigenden Besucherzahlen können sich hier besonders stark positiv wie negativ auswirken.

Höhere Besucherzahlen erfordern häufig mehr Infrastruktur- und Erhaltungsaufwand und verursachen damit Kosten. Der zunehmende Besucherverkehr kann sich, wenn er nicht adäquat entwickelt und gemanagt wird, negativ auswirken und auch zu Verlust der Attraktivität führen. Das Erleben von Natur und Wildnis ist als Massenerlebnis zumeist kaum realisierbar.

Die durch den Tourismus in deutschen Schutzgebieten generierte regionale Wertschöpfung ist beachtlich und kann gerade in infrastrukturell eher benachteiligten naturnahen Räumen einen Beitrag zum Erhalt und zur Entwicklung langfristiger Arbeitsplätze leisten. So belegt eine Studie des Bundesamts für Natur- schutz die regionalökonomischen Effekte des Tourismus im Müritz-Nationalpark und in den Naturparken Hoher Fläming und Altmühltal und zeigt eindeutig deren wirtschaftliche Bedeutung auf: Allein durch den Nationalparktourismus wurden 2004 an der Müritz Bruttoumsätze von rund 13,4 Mio. $€$ erzielt. Dies entspricht dort rund 630 Arbeitsplätzen. Im Naturpark Altmühltal betrugen die Bruttoumsätze durch den Tourismus 20,7 Mio. $€$, im Naturpark Hoher Fläming 6,2 Mio. $€$ (Job et al. 2005).

Naturschutz und Tourismus stehen als Nutzungsformen hier nicht im Konflikt sondern ergänzen einander. Dies soll jedoch nicht darüber hinwegtäuschen, dass die Vereinbarkeit dieser Nutzungen Konzepte und integrative Ansätze benötigt, um die durchaus divergierenden Ansprüche an die Flächennutzung zusammenzubringen und um potenzielle negative Auswirkungen des Tourismus auf Natur und Landschaft zu vermeiden. Dies erfordert neben integrativen Nutzungskonzepten auch die Unterstützung durch nationale und internationale Politiken (s. u.).

Flächennutzungskonkurrenzen und damit verbundene Konflikte können nicht nur zwischen Tourismus und Naturschutz auftreten, sondern auch innerhalb verschiedener touristischer Aktivitäten sowie zwischen Tourismus und weiteren Landnutzungsarten (z. B. der Landwirtschaft). Eine Überlagerung von verschiedenen Freizeitaktivitäten, welche auf eine ähnliche natürliche Ausstattung angewiesen sind, kann zu Konflikten führen. So nutzen beispielsweise Mountainbikefahrer und Wanderer in Mittelgebirgsregionen ähnliche Wege. Insbesondere bei mangelnder Rücksichtnahme können dadurch Nutzungskonflikte auftreten.

Weiterhin können Interessenskollisionen zwischen Tourismus und Landwirtschaft einerseits aus der Aufgabe extensiver Bewirtschaftung mit nachfolgender Aufforstung resultieren. Anderseits können sie durch eine Intensivierung der Bewirtschaftung, etwa im Rahmen des Anbaus von Energiepflanzen, auf Kosten einer attraktiven abwechslungsreich genutzten Kulturlandschaft entstehen. Der Tourismusbranche wird durch einen solchen Nutzungswandel praktisch die Basis entzogen. 


\section{Nationale und internationale Rahmen- bedingungen und Verpflichtungen}

Auf internationaler Ebene hat sich Deutschland im Rahmen der Konvention über die biologische Vielfalt (CBD) auch zur Umsetzung der CBD-Richtlinien für Tourismus und Umwelt verpflichtet. Diese nehmen in allen Stufen des vorgeschlagenen Managementprozesses für einen verantwortungsvollen Umgang im Hinblick auf Tourismus und biologische Vielfalt Bezug auf die Flächennutzung.

Auch auf regionaler Ebene enthalten multilaterale Abkommen und Protokolle Bestimmungen, die die Flächennutzung durch den Tourismus betreffen. So trägt auch das Tourismusprotokoll unter der Alpenkonvention dem sensiblen Ökosystem Rechnung und legt bezüglich der Politik im Beherbergungsbereich fest:

\begin{abstract}
„Die Vertragsparteien entwickeln Politiken im Beherbergungsbereich, die der Begrenztheit des verfügbaren Raumes durch Bevorzugung der kommerziellen Beherbergung und der Erneuerung und Nutzung der bestehenden Bausubstanz sowie durch Modernisierung und Qualitätsverbesserung der bestehenden Beherbergungseinrichtungen Rechnung tragen." (Artikel 11, Tourismusprotokoll der Alpenkonvention [1991]).
\end{abstract}

Die Bundesregierung hat in die im November 2007 vom Kabinett verabschiedete „Nationale Strategie zur Biologischen Vielfalt" nicht nur ein eigenes Kapitel zu naturnaher Erholung und Tourismus aufgenommen, sondern auch explizite Ziele für bestimmte Landschafstypen und Ökosysteme definiert. So formuliert die Strategie das Ziel, die Neuinanspruchnahme von Flächen für Tourismuszwecke in den Alpen und Hochlagen der Mittelgebirge zu reduzieren (BMU 2007).

Diese Konvention sowie die verabschiedeten Protokolle und Strategien eignen sich als Richtschnur für eine Vereinbarkeit von Tourismus und Naturschutz, ihre Umsetzung erfordert jedoch regionalspezifische und individuelle Planungs- und Umsetzungsprozesse.

\section{Umsetzungsinstrument Biosphärenreservate}

Die heute 13 Biosphärenreservate in Deutschland stellen mit insgesamt 1,65 Mio. Hektar repräsentative Natur- und Kulturlandschaften dar, und zählen zu attraktiven Zielen für einen natur- und landschaftsbezogenen Urlaub in Deutschland. Vor dem Hintergrund der skizzierten Auswirkungen des Tourismus auf Natur und Landschaft stehen ökologische Schutzziele gerade in Schutzgebieten häufig in einem Spannungsverhältnis zu touristischen Entwicklungszielen. Biosphärenreservate haben neben der Schutzfunktion zum Zwecke der Erhaltung der biologischen Vielfalt auch die Funktion, eine nachhaltige, wirtschaftliche, soziale und kulturelle Entwicklung zu fördern. So ist in der Sevilla-Strategie des MAB-Programms als Ziel verankert, dass Biosphärenreservate als Modelle für die Landbewirtschaftung und für Ansätze zur nachhaltigen Entwicklung zu nutzen sind. ${ }^{5}$ Darin ist auch der Tourismussektor eingeschlossen, wie in den Kriterien für die Anerkennung von Biosphärenreservaten in Deutschland präzisiert wird: „Der tertiäre Wirtschaftssektor (Dienstleistungen u. a. in Handel, Transportwesen und Fremdenverkehr) soll dem Leitbild einer dauerhaft-umweltgerechten Entwicklung folgen (...) Hier liegen auch die Möglichkeiten für die Entwicklung eines umwelt- und sozialverträglichen Tourismus." (Deutsches MAB-Nationalkomitee 1996, S. 28) Wichtige Aktionsfelder sind dabei vor allem Managementkonzepte, die auf den Grenzen der Belastbarkeit sensibler Räume basieren, sowie die Entwicklung und Umsetzung kooperativer Strategien und integrativer Konzepte, wie sie z. B. im Biosphärenreservat Rhön entwickelt worden sind. So wurde auf Initiative der bayerischen Verwaltungsstelle des Biosphärenreservats Rhön und des ADFC ein Mountainbikingkonzept entwickelt, das sportlich attraktive und gleichzeitig ökologisch weitgehend konfliktfreie Routen ausweist. Und auch der Wanderer kommt auf seine Kosten, wenn sich Mountainbiker an die für sie ausgewiesenen Routen halten. Konflikte zwischen touristischer Nutzung und Naturschutz können so gelöst und alle touristischen Nutzergruppen befriedigt werden (Deutsches MAB-Nationalkomitee 2003).

\section{Anmerkungen}

1) Unter dem Begriff „Parahotellerie“ werden Übernachtungsmöglichkeiten verstanden, die kein Hotel sind (z. B. Ferienwohnungen, Fremdenzimmer, Bauernhöfe). 
2) Vgl. dazu Schliephake 1985.

3) Reliefenergie ist ein Maß, das die Bewegtheit des Geländes erfasst. Es wird bestimmt durch den Höhenunterschied zwischen den höchsten und tiefsten Punkt eines Kartenausschnitts (vgl. dazu Ammer, Pröbstl 1991).

4) Der Flächenanteil der Nationalparke entspricht lediglich 0,54 Prozent des Bundesgebietes.

5) Zum Beispiel: Das Programm „Der Mensch und die Biosphäre" der UNESCO (abgekürzt MABProgramm) setzt sich seit Jahren für integrierte Planungs- und Monitoringmechanismen von wichtigen Ökosystemen und vor allem Biosphärenreservaten ein und fördert den Ausbau des Weltnetzes der Biosphärenreservate (http://www. unesco.de/mab_programm0.html? \&L=0). Die Sevilla-Strategie gibt die Leitlinien für die Entwicklung der Biosphärenreservate vor.

\section{Literatur}

Alpenkonvention, 1991: Protokoll Tourismus; http:// www.cipra.org/documents/14_de/(download 2.9.08)

Ammer, U.; Pröbstl, U., 1991: Freizeit und Natur. Probleme und Lösungsmöglichkeiten einer ökologisch verträglichen Freizeitnutzung. Hamburg

BBR - Bundesamtes für Bauwesen und Raumordnung (Hg.), 2005: Raumordnungsbericht 2005. Berichte, Bd. 21, Bonn

BMU - Bundesministerium für Umwelt, Naturschutz und Reaktorsicherheit, 2007: Nationale Strategie zur Biologischen Vielfalt. Bonn

Bundesamt für Naturschutz, 1997: Biodiversität und Tourismus. Konflikte und Lösungsansätze an den Küsten der Weltmeere. Berlin, Heidelberg

Bundesamt für Naturschutz, 2008: Daten zur Natur. Münster

Büro für Tourismus- und Erholungsplanung, 1994: Arbeitsmaterialien für einen umweltschonenden Tourismus: Touristische Großprojekte. Berlin

Deutsches MAB-Nationalkomitee, 1996: Kriterien für die Anerkennung und Überprüfung von Biosphärenreservaten der UNESCO in Deutschland; http://www. unesco.de/2283.html? \&L=0 (download 2.9.08)

Deutsches MAB-Nationalkomitee, 2003: Voller Leben. UNESCO-Biosphärenreservate - Modellregionen für nachhaltige Entwicklung. Bonn

F.U.R. - Forschungsgemeinschaft Urlaub und Reisen e.V., 2002: Reiseanalyse 2002. Kiel

F.U.R. - Forschungsgemeinschaft Urlaub und Reisen e.V., 2006: Reiseanalyse 2006. Kiel

F.U.R. - Forschungsgemeinschaft Urlaub und Reisen e.V., 2007: Reiseanalyse 2007. Kiel
F.U.R. - Forschungsgemeinschaft Urlaub und Reisen e.V., 2008: Reiseanalyse 2008. Kiel

Job, H. (Hg.), 2008: Die Destination Nationalpark Bayerischer Wald als regionaler Wirtschaftsfaktor. Nationalpark Bayerischer Wald, Wissenschaftliche Reihe. Grafenau

Job, H.; Harrer, B.; Metzler, D. et al., 2005: Ökonomische Effekte von Großschutzgebieten. Untersuchung der Bedeutung von Großschutzgebieten für den Tourismus und die wirtschaftliche Entwicklung der Region. BfN-Skripten 135. Bonn

Krumrey, N.; Märker, J., 2005: Freizeit- und Erlebnisparks: Umweltauswirkungen; Lösungsansätze; Handlungsempfehlungen. Studienarbeit im Fach Ökologie und Tourismus - Freizeit und Umwelt, Umweltökonomie in Freizeit und Tourismus an der Hochschule Bremen. Unveröffentlicht

Kuckartz, U.; Rädiker, S.; Rheingans-Heintze, A., 2006: Umweltbewusstsein in Deutschland 2006. Ergebnisse einer repräsentativen Bevölkerungsumfrage.Marburg

Schliephake, K., 1985: Fremdenverkehr und Flächenkapazität - zur Frage von Flächenbelastung und Flächenverbrauch durch den Erholungstourismus. In: Arbeitskreise zur Landentwicklung in Hessen der ASG (Hg.): Landeignung und Bodenschutz, S. $175-205$

Statistisches Bundesamt, 2007: Statistisches Jahrbuch 2007. Wiesbaden

Statistisches Bundesamt, 2008: Ankünfte und Übernachtungen von Gästen in Beherbergungsstätten und auf Campingplätzen. Zeitreihe; http://www. destatis.de/jetspeed/portal/cms/Sites/destatis/Inter net/DE/Content/Statistiken/Binnenhandel/Touris mus/Tabellen/Content75/AnkuenfteCamping plaetze.psml (download 29.7.08)

Umweltbundesamt, 2002: Umwelt und Tourismus. Daten, Fakten, Perspektiven. Berlin

\section{Kontakt}

Dipl.-Biol. Barbara Engels

Bundesamt für Naturschutz

Fachgebiet „Gesellschaft, Nachhaltigkeit, Tourismus und Sport"

Konstantinstr. 110, 53179 Bonn

E-Mail: Barbara.Engels@bfn.de

Internet: http://www.bfn.de 